\title{
The Marconi Challenge: Who Needs the IEEE MicroMouse?
}

\author{
Dennis Silage \\ Electrical and Computer Engineering \\ College of Engineering, Temple University
}

\begin{abstract}
Presented here from experience is a challenging new competition to supplement the IEEE MicroMouse contest. The IEEE MicroMouse contest has a storied history but is staid, technically complicated and expensive to mount. The Marconi Challenge is a new contest that addresses the design objectives of wireless data communication and is suitable for students from junior high school to college. The Marconi Challenge was originally conceived to celebrate the 100th anniversary of Guglielmo Marconi's transatlantic wireless transmission in 2001. For high school students, the Marconi Challenge provides an opportunity to demonstrate the principles of lens and mirrors in optics and basic electronics in a stimulating environment. The transmission medium is infrared light and the requisite components and test equipment are inexpensive, easily obtained and no license is required. Complete teaching modules have been developed and accepted by a school district for use in the junior high school science and senior high school physics curriculum. Undergraduate ECE students can utilize more complex electronics, error correcting codes and RF modulation methods with an Amateur Radio license to explore applications of wireless communication based on their curriculum.
\end{abstract}

\section{The Long Road to the IEEE MicroMouse}

The IEEE MicroMouse contest, in which an autonomous robotic vehicle negotiates a geometric maze, traces its roots to a 'mouse versus maze' problem using relay logic that was proposed in 1950 at MIT. It first debuted in 1979 with 15 microprocessor-controlled entries, only 4 of which managed to solve the 8 by 8 -foot maze. The IEEE MicroMouse contest continues today with either the original rules and the same design objectives or variations, such as the ASEE Model Design Competition.

Temple University (TU) has provided an entry and hosted the MicroMouse contest sponsored by the Philadelphia Section of the IEEE. A team of TU ECE undergraduates recently developed an innovative MicroMouse entry. Rather than utilize a commercial autonomous robotic vehicle, the TU ECE MicroMouse used an Intel i386EX 32-bit embedded processor in a microcomputer which was interfaced to custom analog and digital electronics. The process control and mazesolving software was written in $\mathrm{C}$ and assembly language and tested on a smaller quadrant maze before the actual competition.

The MicroMouse traditionally uses two wings equipped with a linear array of infrared (IR) transmitters and receivers. The wings ride over the top of the maze by design and provide a navigational signal for the MicroMouse to traverse the corridors of the arbitrary contest maze. 
However, at the intersections of the maze one or both of the linear IR sensor arrays have no signal and the MicroMouse may attempt to affect a 90 degree turn without any feedback of its
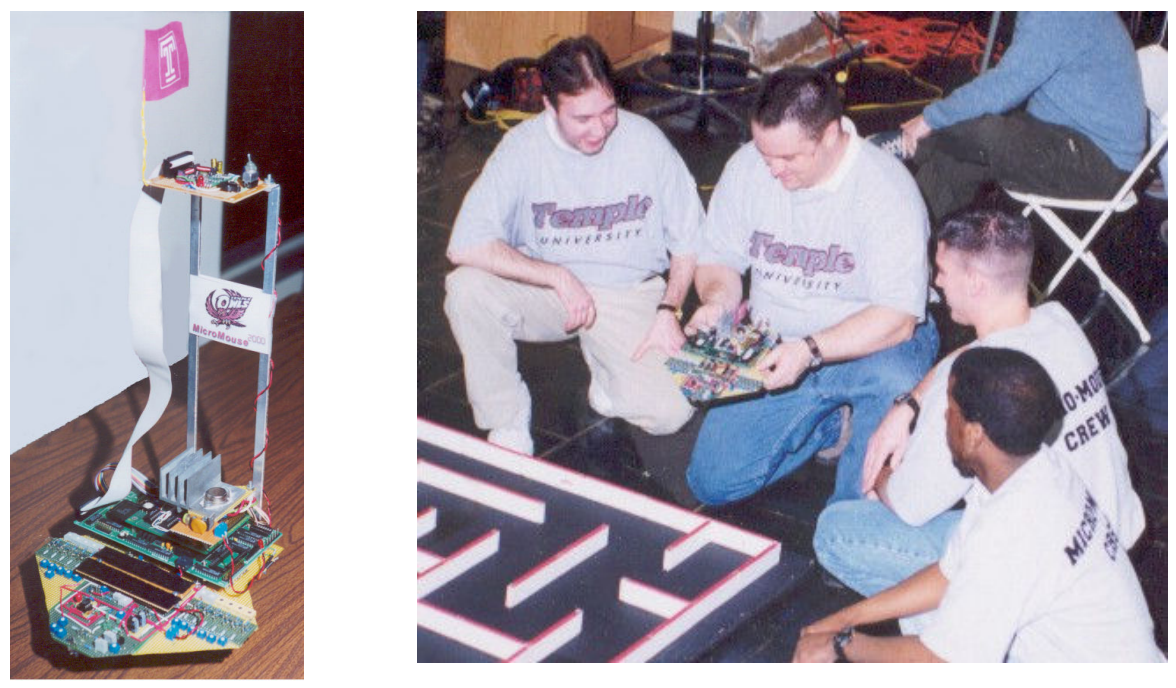

Temple University ECE MicroMouse (2001) and the undergraduate team in the IEEE Philadelphia Section regional contest (2000).

orientation. The TU ECE MicroMouse entry in 2000 turned by counting the number of commands sent to its wheels. Unfortunately, the wheels notoriously slip which causes the turn to be incomplete. The MicroMouse can recover once its wings reacquire an IR sensor signal from the top of the next maze wall, but this takes valuable time. The 2001 TU ECE MicroMouse added an electronic magnetometer compass that provided the exact orientation of the device as it turned.

The results were sharply executed, complete turns and a contest winner, but then what? What follows a successful entry in the MicroMouse contest? Other than as spectators, participation by a large cadre of junior and senior high school students also seems to be missing.

The MicroMouse contest has been in existence for over 25 years and its design objectives, and often solutions, are well documented. Commercial robotic devices for the MicroMouse contest are available, but are somewhat expensive. The complexity and requisites of the contest, including the maze itself, seems to limit the incentive to organize a MicroMouse event. The traditional IEEE MicroMouse contest is now often supplanted by other semiautonomous robotic vehicles, such as the Lego robots, and organized events such as the Fire-Fighting Home Robot Contest, but the expensive and effort to be a participant and to mount an event remains prohibitive.

\section{See the Light!}

The Marconi Challenge is a new contest that addresses the design objectives of wireless data communication and is suitable for students from junior high school to college. The Marconi Challenge was originally conceived to celebrate the 100th anniversary of Guglielmo Marconi's 
transatlantic wireless transmission in 2001. In 1901 Marconi succeeded in transmitting a radio signal across the Atlantic Ocean. The wavelength of the transmitted signal was approximately 1500 meters, and input power was measured in kilowatts. In contrast, the Marconi Challenge requires that junior and senior high school participants transmit an infrared (IR) light signal at a wavelength of 940 nanometers and power measured in milliwatts to a receiver at the greatest discernable distance from the transmitter.

The Marconi Challenge offers unique opportunities for experimentation and learning for junior and senior high school students. IR digital data communication is used to provide an educational experience in electronics, the use of semaphores to represent information, and the transmission and reception of IR light. Unlike low power, unlicensed radio frequency (RF) transmissions, whose electronic circuitry is more complicated, IR semiconductor components are inexpensive and the circuitry is easy to comprehend, construct and utilize.

In a junior and senior high school educational module, the students are first instructed on the simple principles of the IR light emitting diode (LED) transmitter and IR photodetector receiver. The Marconi Challenge competition entry form prescribes a general configuration for the IR light communication system, specifications for the electronic components and a schematic. A complete bill of materials, principles of the technology, construction details and the rules of the Marconi Challenge are also provided. Standard components for the Marconi Challenge include a single high-output IR LED as a transmitter, and an IR phototransistor as a detector.
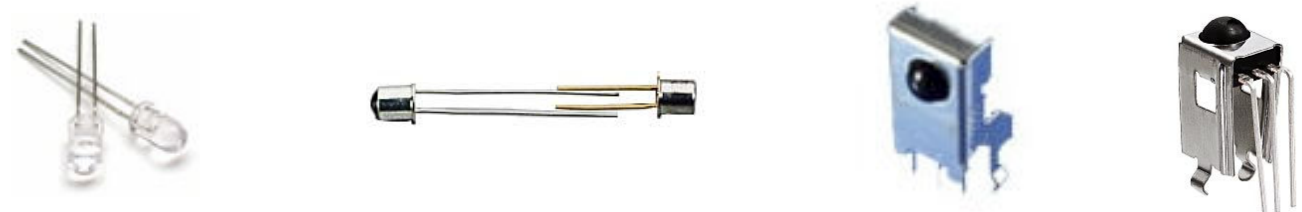

IR LED transmitters and IR phototransistor detectors as specified for the Marconi Challenge.

Common passive components are also used in the construction of the IR transmitter and receiver. Both the active IR and passive components are available ay various retail electronic outlets, such as Radio Shack.

The IR receiver has an acoustic sounder to verify the presence of a signal. The students are given the electronic schematic for the IR transmitter and receiver and instructed on the principles of fabrication, including soldering. The students lay out the parts on a common perforated circuit board and solder them into place. This exercise introduces the relationship between an electronic schematic and a physical layout with components.

The Marconi Challenge contest rules, modified every year, have limited the IR transmitter current to a maximum value, which is verified. The entry form also suggests experiments, using Ohm's law and measurements, to set the transmitter current and, by varying the collector load resistor, adjusting the sensitivity of the phototransistor. The students learn that there are operating points that will optimize the IR transmitter LED and IR receiver phototransistor. 
The Marconi Challenge competition requires only the basic tools of soldering iron, solder, cutting and needle nose pliers and disposable electronic and passive components. A volt-ohm meter is needed to measure circuit currents and voltages.

The IR light transmission of information introduces the concepts of optics in an interesting manner. Focal lengths of standard physics lab one to four-inch convex lenses and mirrors are measured and stable lens mounts are constructed by the students. Ray tracing predicts where the IR light transmission should be focused and this is verified experimentally as part of the education module before the competition. The rules of the Marconi Challenge allow physically modifying the half-intensity angle of the IR LED, which is nominally 45 degrees. The performance of different IR LED-lens and lens-phototransistor configurations is compared. Any method, except fiber optics, can be used at the IR receiver to focus and capture the optical transmission.

The junior and senior high school students are next introduced to the important concept of semaphores that are used to communicate. Initially, the Morse code is employed. The students code, send and decode simple messages. Rather than memorized the Morse code, the intrinsic difference in the sound of the transmission is emphasized. For example, the difference in the sound between YES and NO can convey the meaning of the transmission. Interested students have continued their studies in Morse code, electronics, and communication regulations and have taken the examination for an Amateur Radio license. Amateur Radio is an interesting way to entice high school students to consider the ECE profession.

\section{From Marconi to Amateur Radio}

For undergraduate ECE students, more complex passband amplitude (ASK), frequency (FSK) or phase shift keying (PSK) digital modulation and demodulation, frequency hopping (FHSS) and discrete sequence spread spectrum (DSSS) and error correction techniques are detailed in the yearly Marconi Challenge contest rules. These entries use either low power unlicensed RF transmissions on 300 or $432 \mathrm{MHz}$ but, preferably, high power Amateur Radio licensed microwave transmissions on 1.2, 2.4, 5.6 and 10 GHZ. Amateur Radio has been used extensively in the TU ECE capstone senior design project and it's certainly not just for Morse code anymore ${ }^{1}$.

The Marconi Challenge can also be integrated into the ECE capstone senior design course. The active components include RF ICs and microwave transistors, $10 \mathrm{GHz}$ Gunn diodes, and IR or laser transmitters and receivers. Microwave stripline electronic circuit designs and helix and horn antennas incorporate undergraduate ECE course work in electromagnetic wave propagation.

Embedded processors using microprocessors or programmable gate arrays (PGA) are used for source encoding and decoding, bit time synchronization and error correction and incorporate undergraduate ECE course work in digital logic and processor systems. The technical challenges and contest metrics for undergraduate ECE students include outdoor data transmissions and bit error rate (BER) measurements.

A recent undergraduate ECE student entry is shown below, which utilizes an Atmel AVR microprocessor to amplitude shift key (ASK) the IR light data transmission and apply a $(7,11)$ 
Hamming block code for error correction. A C mount lens focuses the IR light and improves the optical channel.

More details of the Marconi Challenge can be found on the website of the Temple University Amateur Radio Club (TUARC, K3TU) which sponsors the competition for junior and senior high school students: www.temple.edu/k3tu/marconi.htm
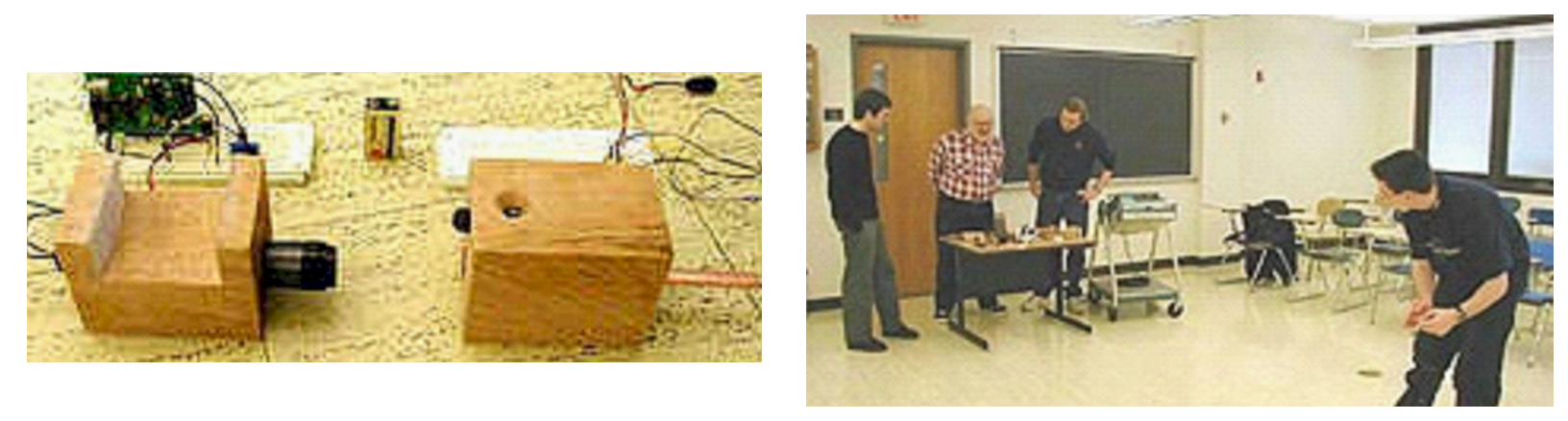

Undergraduate ECE student entry in the Marconi Challenge and the indoor competition.

\section{A Contest that Resonates with the Wireless World}

IR data transmission projects are much simpler to comprehend and less expensive to build than are robotics projects for junior and senior high school students. The Marconi Challenge has been expanded for the pre-College student to include electronic circuits for PC serial-port interfacing and ASCII character data transmission. Improved optics, telescopes and parabolic mirrors have allowed IR data communication across the width of an entire high school ground. The Marconi Challenge seems to resonate better with our wireless world!

1. Dennis Silage, Reintroducing Amateur Radio In ECE Capstone Design Projects, Proceed. 2004 ASEE Annual Conf.

DENNIS SILAGE (silage@temple.edu) received the PhD in EE from the University of Pennsylvania in 1975. He is a Professor, teaches digital data communication and digital signal processing and is the trustee of the Temple University Amateur Radio Club (K3TU, www.temple.edu/k3tu), which he has integrated into the undergraduate communications curriculum. Dr. Silage is a past chair of the Middle Atlantic Section of the ASEE. 